OPEN ACCESS

Edited by:

Valerio Carelli,

University of Bologna, Italy

Reviewed by:

Michael S. Vaphiades,

University of Alabama at Birmingham,

United States

Yanchun Ji,

Zhejiang University, China

Vittoria Petruzzella,

University of Bari Aldo Moro, Italy

Julio Montoya,

University of Zaragoza, Spain

*Correspondence:

Zhe Liu

doctorliuzhe@126.com

Specialty section:

This article was submitted to

Neuro-Ophthalmology,

a section of the journal

Frontiers in Neurology

Received: 31 August 2020

Accepted: 10 February 2021

Published: 04 March 2021

Citation:

Sun $C B$, Bai $H X, X u D N, X i a o Q$ and Liu Z (2021) Mitochondrial 13513G>A

Mutation With Low Mutant Load

Presenting as Isolated Leber's

Hereditary Optic Neuropathy

Assessed by Next Generation

Sequencing.

Front. Neurol. 12:601307.

doi: 10.3389/fneur.2021.601307

\section{Mitochondrial 13513G >A Mutation With Low Mutant Load Presenting as Isolated Leber's Hereditary Optic Neuropathy Assessed by Next Generation Sequencing}

\author{
Chuan-bin Sun ${ }^{1}$, Hai-xia Bai ${ }^{1}$, Dan-ni Xu ${ }^{1}$, Qing Xiao ${ }^{1}$ and Zhe Liu ${ }^{2 *}$ \\ ${ }^{1}$ Eye Center, Second Affiliated Hospital of Zhejiang University School of Medicine, Hangzhou, China, ${ }^{2}$ Department of \\ Ophthalmology, Zhejiang Provincial People's Hospital, People's Hospital of Hangzhou Medical College, Hangzhou, China
}

Objective: Mitochondrial 13513G > A mutation presenting as isolated Leber's hereditary optic neuropathy (LHON) without any extraocular pathology has not been reported in literature. We herein evaluate the clinical characteristics and heteroplasmy of m.13513G $>$ A mutation manifesting as isolated LHON.

Methods: Seven members of a Chinese family were enrolled in this study. All subjects underwent detailed systemic and ophthalmic examinations. Mitochondrial DNA in their blood was assessed by targeted PCR amplifications, next generation sequencing (NGS), and pyrosequencing. One hundred of blood samples from ethnic-matched healthy volunteers were tested by NGS and pyrosequencing as normal controls.

Results: Isolated LHON without any other ocular or extraocular pathology was identified in a 16 year old patient in this family. Heteroplasmic m.13513G > A mutation was detected by NGS of the full mtDNA genome in the patient with mutant load of $33.56 \%$, and of $26 \%$ 3 months and 3 years after the onset of LHON, respectively. No m.13513G $>$ A mutation was detected in all his relatives by NGS. Pyrosequencing revealed the mutant load of m.13513G > A mutation of the LHON patient, his mother, father and sister were 22.4, 1.9, 0 , and $0 \%$, respectively. None of 100 healthy control subjects was detected to harbor m.13513G > A mutation either by NGS or by pyrosequencing of the full mt DNA genome.

Conclusions: We first report m.13513G>A mutation with low mutant load presenting as isolated LHON. NGS of the full mitochondrial DNA genome is highly recommended for LHON suspects when targeted PCR amplification for main primary point mutations of LHON was negative.

Keywords: Leber's hereditary optic neuropathy, mitochondrial DNA, gene mutation, m13513G>A, optic atrophy

\section{INTRODUCTION}

As one of the most common mitochondrial inherited diseases, Leber's hereditary optic neuropathy (LHON) is typically characterized by acute painless bilateral central vision loss in adolescents and young adults, predominantly in males (1-4). Previous investigations have revealed that more than $90 \%$ of LHON cases are related to one of three primary point mutations in the mitochondrial 
DNA (mtDNA): m.11778G $>$ A, m.3460G $>$ A, and m.14484T $>$ C, which encode the ND4, ND1, and ND6 subunits of Complex I in the mitochondrial respiratory chain, respectively (5-7). However, other rare primary mtDNA mutaions such as $\mathrm{m} .3635 \mathrm{G}>\mathrm{A}$ in ND1, m.14495A $>$ G in ND6, and m.13513G $>$ A in ND5 have also been reported to cause LHON independently (1-7).

Since first identified as a gene mutation causative for mitochondrial encephalomyopathy with lactic acidosis and stroke-like episodes (MELAS), m.13513G $>$ A mutation has been mostly reported in Leigh syndrome (LS), MELAS, as well as MELAS/LHON and MELAS/LS overlap syndromes $(5,6)$. Ophthalmic manifestations related to $\mathrm{m} \cdot 13513 \mathrm{G}>\mathrm{A}$ mutation including optic atrophy, ptosis, and chronic progressive external ophthalmoplegia (CPEO) are mostly accompanied by MELAS or LS, and classified as MELAS /LHON, LS/LHON, or MELAS/CPEO overlap syndromes (5-8).

Until now, m.13513G $>$ A mutation-related LHON without LS or MELAS has been reported in only three cases (9-11). However, all above 3 cases were accompanied by other extraocular pathology such as hypertrophic myocardiopathy, myopathy, Wolff-Parkinson White syndrome, chronic kidney disease, diabetes mellitus, or hearing loss. To our knowledge, m.13513G $>$ A mutation presenting as isolated LHON without any extraocular pathology has not been reported. We herein evaluate the clinical characteristics and heteroplasmy of the m.13513G $>$ A mutation manifesting as isolated LHON without any other ocular or extraocular pathology.

\section{METHODS}

\section{Subjects and Clinical Examinations}

Six maternal family members (including one half-brother and one half-sister) and the farther of the LHON patient of Han population in a Chinese family were enrolled in this study (Figure 1). Detailed medical records of the patient visiting different hospitals were collected. All subjects underwent detailed systemic and ophthalmic examinations including consciousness, hearing, articulation, superficial and deep sensation, muscle strength, tone and reflexes, best corrected visual acuity (BCVA), slit-lamp microscopy, color fundus photography, and visual field test, as well as blood test for cardiac enzymes, blood lactate fluctuation during exercise test, electrocardiogram and Doppler echocardiography. Gadolinium enhanced orbital MRI, and magnetic resonance spectroscopy were performed only in the LHON patient.

\section{Mitochondrial DNA Assessment}

Peripheral blood was collected from the patient and his relatives for mtDNA sequencing after informed consents were signed. One hundred of blood samples from ethnicmatched healthy volunteers were used as normal controls. Targeted PCR amplifications and Sanger DNA sequencing of three main primary point mutations of LHON in $\mathrm{mt}$ DNA, i.e.: m.11778G $>$ A, m.3460G $>A$, and m.14484T $>C$, were first assessed in the LHON patient according to procedures described previously $(12,13)$. In brief, QIAamp
DNA Blood Mini kits were employed for genomic DNA extraction (Qiagen, NO.51104). Three main primary mutations of LHON including m.11778G $>A$, m.3460G $>A$, and m.14484T $>C$ were assessed by targeted PCR amplifications of DNA fragments, using oligodeoxynucleotides that correspond to mitochondrial DNA at 11,654-11,865 for m.11778G $>$ A mutations; 3,108-3,717 for m.3460G $>$ A mutations, and 14,260-14,510 for m.14484T $>C$ mutations (12). Fragments were isolated and analyzed in an ABI 3700 automated DNA sequencer (Applied Biosystems; Thermo Fisher Scientific, Inc.) using Big Dye Terminator Cycle sequencing kits. Comparisons of the fragments were performed through Cambridge sequencing (Gen-Bank accession number: NC_012920).

Since none of above three primary point mutations in $\mathrm{mt}$ DNA was detected in the LHON patient. Next generation sequencing (NGS) of the whole mtDNA genome and DNA sequence analysis were then performed in the patient based on procedures described previously $(14,15)$. In brief, whole genomic DNA was extracted with the Purgene DNA isolation kit (Qiagen), and the entire genome of mitochondrial was amplified total of three pooled reactions by PCR as 99 separate fragments, using primers for the light and heavy stranded DNA. Then the DNA was purified using magnetic beads and a library was made for directly sequenced on a sequencer using a NGS reaction kit (MiniSeq, Illumina). To identify mutations within the obtained genome, the consensus Cambridge sequence (Gen-Bank accession number: NC_012920) was used as a reference $(14,15)$. To detect m.13513G $>A$ mutation in the mitochondrial ND5 gene in the patient, his relatives and normal controls, 13,319-14,287 region was amplified using: forward 5'-ACA TCT GTA CCC ACG CCT TC-3, and reverse 5' - AGA GGG GTC AGG GTT GAT TC-3', as described previously $(12,14)$, and analyzed as mentioned in the context.

To further detect and quantify the mutant load of the m.13513G4A mutation in the LHON patient, his relatives and normal controls, specific single nucleotide polymorphism assays using pyrosequencing which is more sensitive in detecting low-level DNA mutations were performed as described previously $(16,17)$. In brief, PCR amplification of a mtDNA fragment containing the m.13513 position was carried out on Pyrosequencer PSQ96MA platform using the following primers: forward 5'-CTTCAACCTCCCT CACC-3', reverse 5'AGCGCTGCTCCGGTTCATAGATTGCTCAGGCGTTT GTG TATGA-3', and sequencing A(G/A)ACCACAT (nt. 1351213520). Sequence identification was performed by the PSQ SQA software (Biotage AB), and the percentage of mutant load was determined using the quantification function of the software.

Mitochondrial DNA sequences of 17 vertebrates were assessed for interspecific analysis $(13,18)$. The confidence interval (CI) was measured through the comparison of human nucleotide variants to other vertebrates $(n=16)$. The $\mathrm{CI}$ indicates the percentage of species with wild type nucleotides at an identical position. The mitochondrial haplogroups of the Asian were also determined as described previously $(19,20)$. 


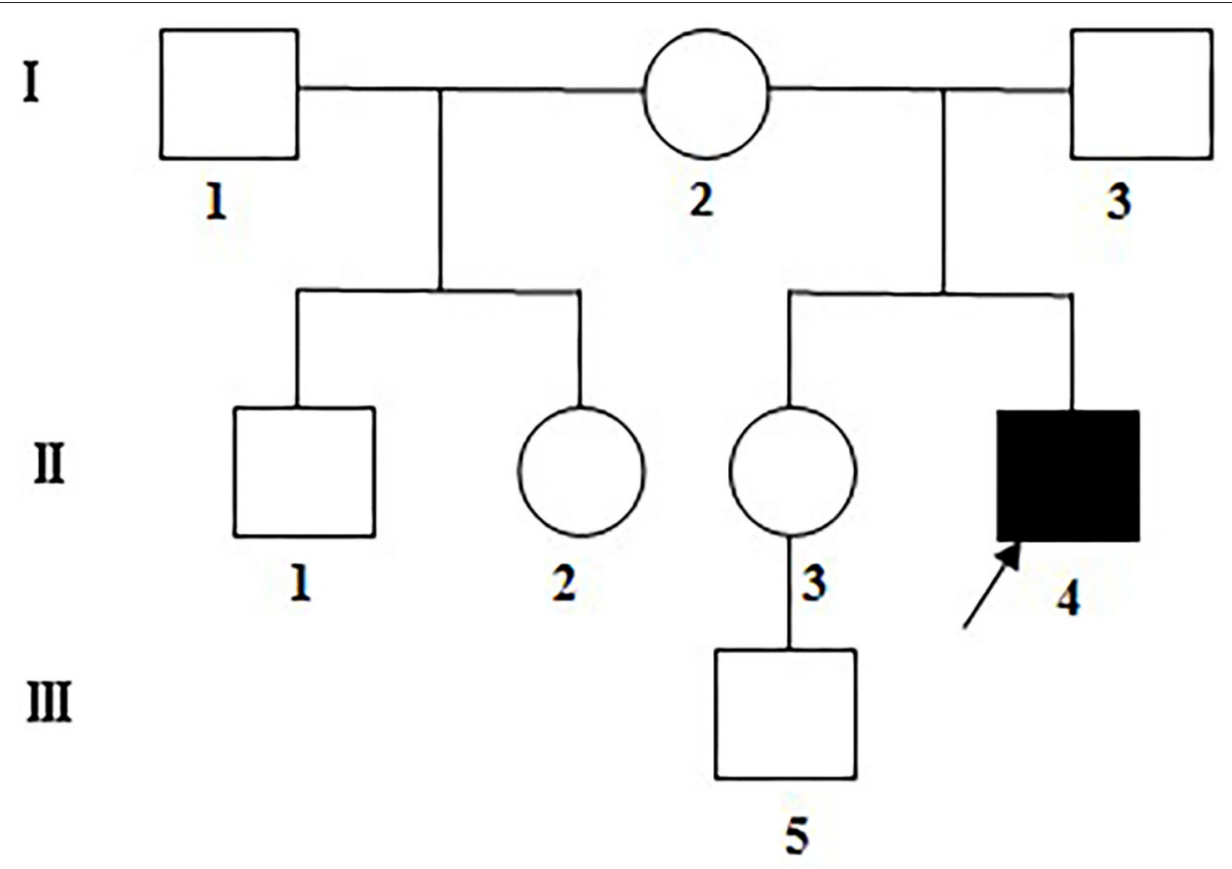

FIGURE 1 | The Chinese LHON family under assessment. Visually impaired family members: filled symbols; females: circles; males: squares; arrow: the proband.

\section{RESULTS}

\section{Clinical Characteristics of $\mathrm{m} .13513 \mathrm{G}>\mathrm{A}$ Mutation-Related LHON}

Isolated LHON without any other ocular or extraocular pathology was identified in one patient in this family. The patient was a 16 years old male presenting with a complaint of sequential painless bilateral central vision loss. He first experienced a blurred vision in the left eye, unfortunately he did not pay any attention until another sudden vision loss in the right eye 1 month later, he was then taken to the local hospital for ophthalmic examination. Medical record revealed a BCVA of $20 / 100$ and counting fingers in the right and left eye, respectively, fundus photography revealed congested optic disc edema in the right eye, and pale temporal optic disc with congested nasal optic disc edema in the left eye (Figures 2A,B). Octopus visual field test showed large centrocecal scotoma in the right eye and diffuse field constriction in the left eye (Figures 2C,D).

His past medical history was unremarkable, and he denied any other ocular or extraocular symptoms such as ptosis, extraocular muscle paralysis, sensation or movement disorders, epilepsy episode, or hearing loss. His physical examination was normal. Blood test for basic metabolic panel was unremarkable. Gadolinium enhanced orbital MRI examination showed normal signals of bilateral optic nerves and brain. He was initially diagnosed as bilateral idiopathic demyelinating optic neuritis, and treated with intravenous methylprednisolone pulse therapy $(500 \mathrm{mg}$ qd $\times 3 \mathrm{~d})$ followed by gradual tapering of oral methylprednisolone. Unfortunately, the patient showed no response to steroid therapy, and his vision progressively deteriorated in both eyes.
When the patient first came to our eye center another 2 weeks later, his vision was counting fingers in the right and hand motion in left eye, pupil size was $5 \mathrm{~mm}$ and pupillary light reflex was sluggish in both eyes with a relative afferent pupil defect in the left eye, and temporal optic disc was pale in both eyes (Figures 3A,B). Blood test for neuromyelitis optica-IgG antibody and myelin oligodendrocyte glycoprotein antibody were both negative. Bilateral LHON was suspected and mtDNA assessment was highly recommended. The patient initially refused our suggestion, but finally accepted and underwent mtDNA assessment 1 month later when he visited another neuroophthalmologist in another hospital and was clinically diagnosed as LHON.

None of three main primary point mutations in mtDNA related to LHON was detected by targeted PCR amplifications and Sanger DNA sequencing in the patient. However, heteroplasmic m.13513G $>$ A mutation was detected by NGS and pyrosequencing of the full $\mathrm{mt}$ DNA genome in this paient (Figures 4A,B). Hence, his diagnosis was corrected to m.13513G $>$ A mutation-related LHON.

To further exclude other possible mitochondrial diseases, the patient was referred to a neurologist and a cardiologist for consultation. However, his neurological examinations were all normal including consciousness, hearing, articulation, superficial and deep sensation, as well as muscle strength, tone, and reflexes. Blood test for cardiac enzymes including creatine kinase and lactate dehydrogenase were both unremarkable. Blood lactate fluctuation was within normal limits during exercise test. Electrocardiogram was normal and Doppler echocardiography revealed normal cardiac morphology and hemodynamics. There 

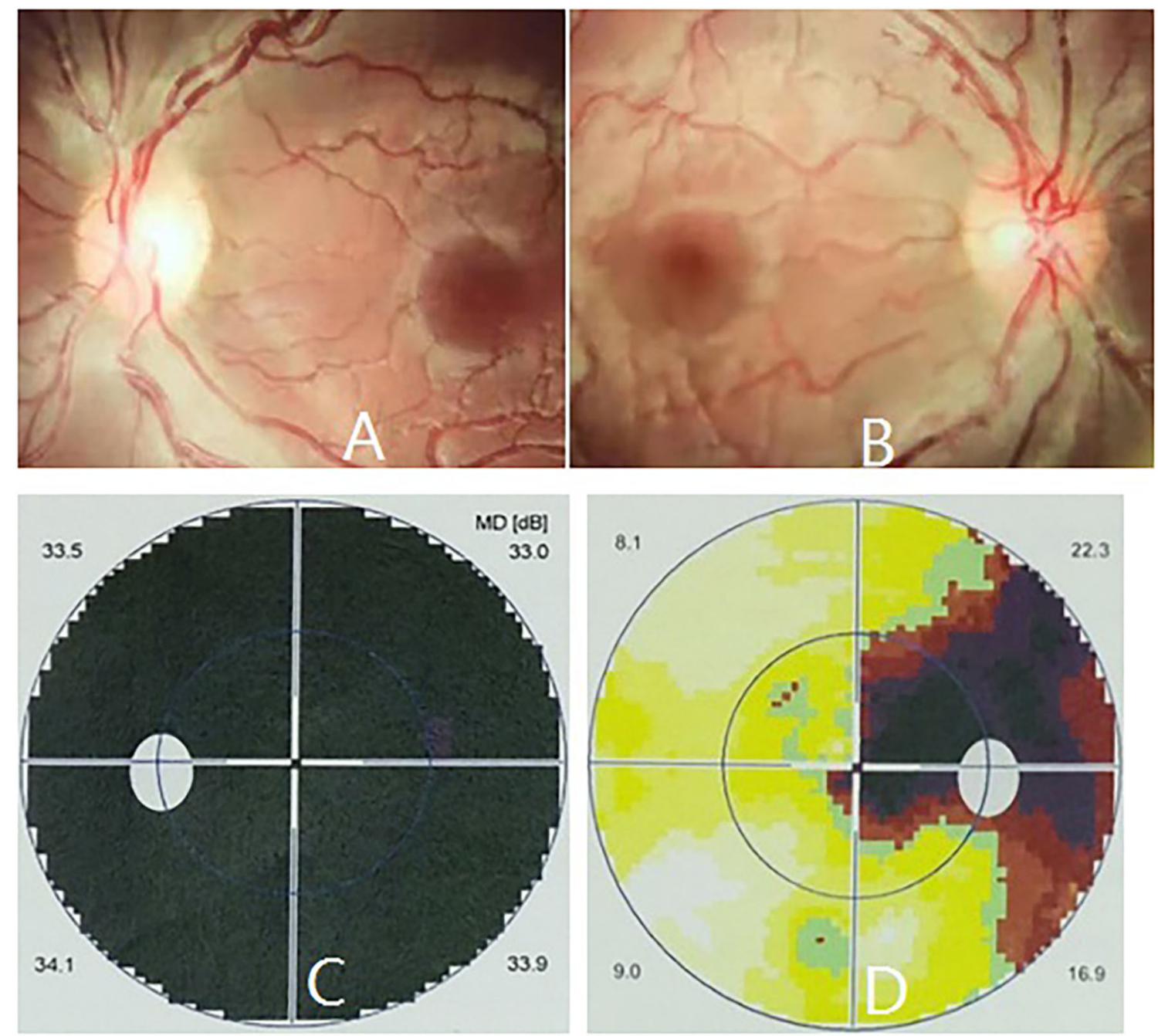

FIGURE 2 | Ophthalmic manifestations of m.13513G>A mutation-related LHON at presentation. Fundus photography revealed pale temporal optic disc with congested nasal optic disc edema in the left eye (A), and congested optic disc edema in the right eye (B), respectively. Octopus visual field test showed full field blindness in the left eye (C) and large centrocecal scotoma in the right eye (D), respectively.

was no lactic acid peak found in the brain by magnetic resonance spectroscopy.

The patient was regularly followed up for 4 years, ophthalmic examination at last follow-up revealed spontaneously slight increase in his vision (20/320 in the right eye and counting fingers in the left eye), and pale optic disc in both eyes (Figures 3C,D). However, no other ocular or extraocular pathology was found during his follow-up.

Although all relatives of the patient underwent detailed systemic and ophthalmic examinations, no abnormal findings in consciousness, hearing, articulation, superficial and deep sensation, muscle strength, tone, and reflexes, BCVA, anterior segment and fundus examination, visual field test, as well as blood test for cardiac enzymes, blood lactate fluctuation during exercise test, electrocardiogram and Doppler echocardiography, were found during 4 years' follow-up.

\section{Mitochondrial DNA Assessment of m.13513G > A Mutation-Related LHON}

None of three main primary point mutations of LHON, i.e., m.11778G $>$ A, m.3460G $>$ A, and m.14484T $>$ C, were detected by targeted PCR amplifications and Sanger DNA sequencing in the patient. However, heteroplasmic m.13513G $>$ A mutation in the mitochondrial ND5 gene was detected by NGS of the full mtDNA genome and further confirmed by Sanger DNA sequencing in the patient (Figure 4A).

Mutant load of $\mathrm{m} .13513 \mathrm{G}>\mathrm{A}$ mutation in the patient assessed by NGS was $33.56 \%$ (Mygenostics Company, Beijing, China) 3 months after the onset of bilateral vision loss, and $26 \%$ (Amplicon Gene Company, Shanghai, China) 3 years after the onset of bilateral vision loss. Nevertheless, no m.13513G $>$ A mutation was detected in all his relatives by either of above twice NGS tests. 

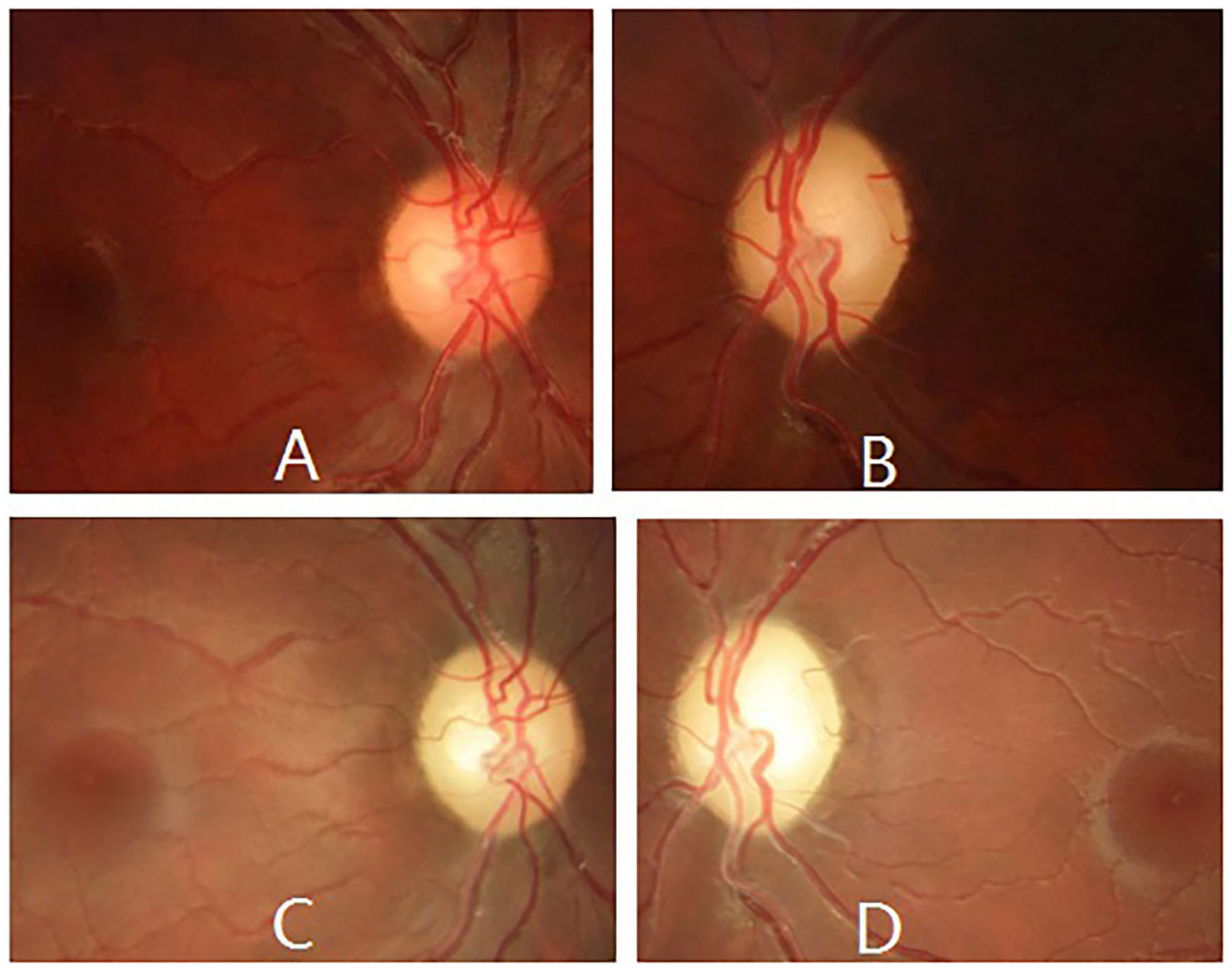

FIGURE 3 | Ophthalmic manifestations of m.13513G>A mutation-related LHON during follow-up. Fundus photography revealed temporal optic disc was pale in both eyes 2 weeks later $(\mathbf{A}, \mathbf{B})$, and pale optic disc in both eyes 4 years later $(\mathbf{C}, \mathbf{D})$.

Pyrosequencing of the full mt DNA genome was assessed 3 years after the onset of bilateral vision loss in this patient, and m.13513G $>$ A mutation with a mutant load of $22.4,1.9,0$, and $0 \%$ was detected in the patient, his mother, father and other maternal relatives, respectively (Figure 4B). None of 100 healthy control subjects were detected to harbor m.13513G $>$ A mutation either by NGS or by pyrosequencing of the full $\mathrm{mt}$ DNA genome.

Based on crystal-structures of complex I in mammals, m.13513 encoded the residue D393 of ND5 protein (Figure 5). This residue was found to be highly conserved amongst ND5 proteins of the 17 organisms. The array of polymorphic loci in the mitochondrial DNA of the family investigated in this study was shown in Table 1, there were 2 variants detected in $12 \mathrm{~S}$ rRNA gene, 2 variants in $16 \mathrm{~S}$ rRNA gene, 11 variants in D-loop, one variant in tRNA, and 9 missense mutations and 17 silent variants in protein- encoding genes. The phylogeny of variants were investigated through comparisons to mice (21), cows (22), and Xenopus laevis (23), whereas none of them showed obvious evolutionary conservation. Moreover, according to the nomenclature of mitochondrial haplogroups, these mtDNA polymorphic variations in this pedigree belonged to the Eastern Asian haplogroups D4a.

\section{DISCUSSION}

Although $\sim 90 \%$ of the mtDNA mutations related to LHON was one of three primary mutations, i.e., m.11778G $>$ A, m.3460G $>$ A, and $\mathrm{m} .14484 \mathrm{~T}>\mathrm{C}$, other rare primary mtDNA mutaions including $\mathrm{m} .13513 \mathrm{G}>\mathrm{A}$ have also been reported to cause LHON independently (1-7). As the most frequently reported mutation in the mitochondrial ND5 gene, m.13513G > A mutation was identified as a causative gene mutation mostly related to LS, MELAS, and LS or MELAS related overlap syndromes $(7-11,24,25)$. Until now, LHON caused by m.13513G $>$ A mutation yet not accompanied by LS or MELAS was reported in only three cases (9-11). However, all above three LHON cases were accompanied by other ocular and (or) extraocular pathology (9-11). To our knowledge, this is the first report of the m.13513G $>$ A mutation presenting as isolated LHON yet without any other ocular or extraocular pathology.

In mitochondrial diseases, it is not unusual for the same mutation in the same gene to result in different manifestations. Previous study indicated that germ-line mtDNA bottleneck existed during oogenesis and caused significant heteroplasmy frequency shifts between generations. As for a pathogenic 

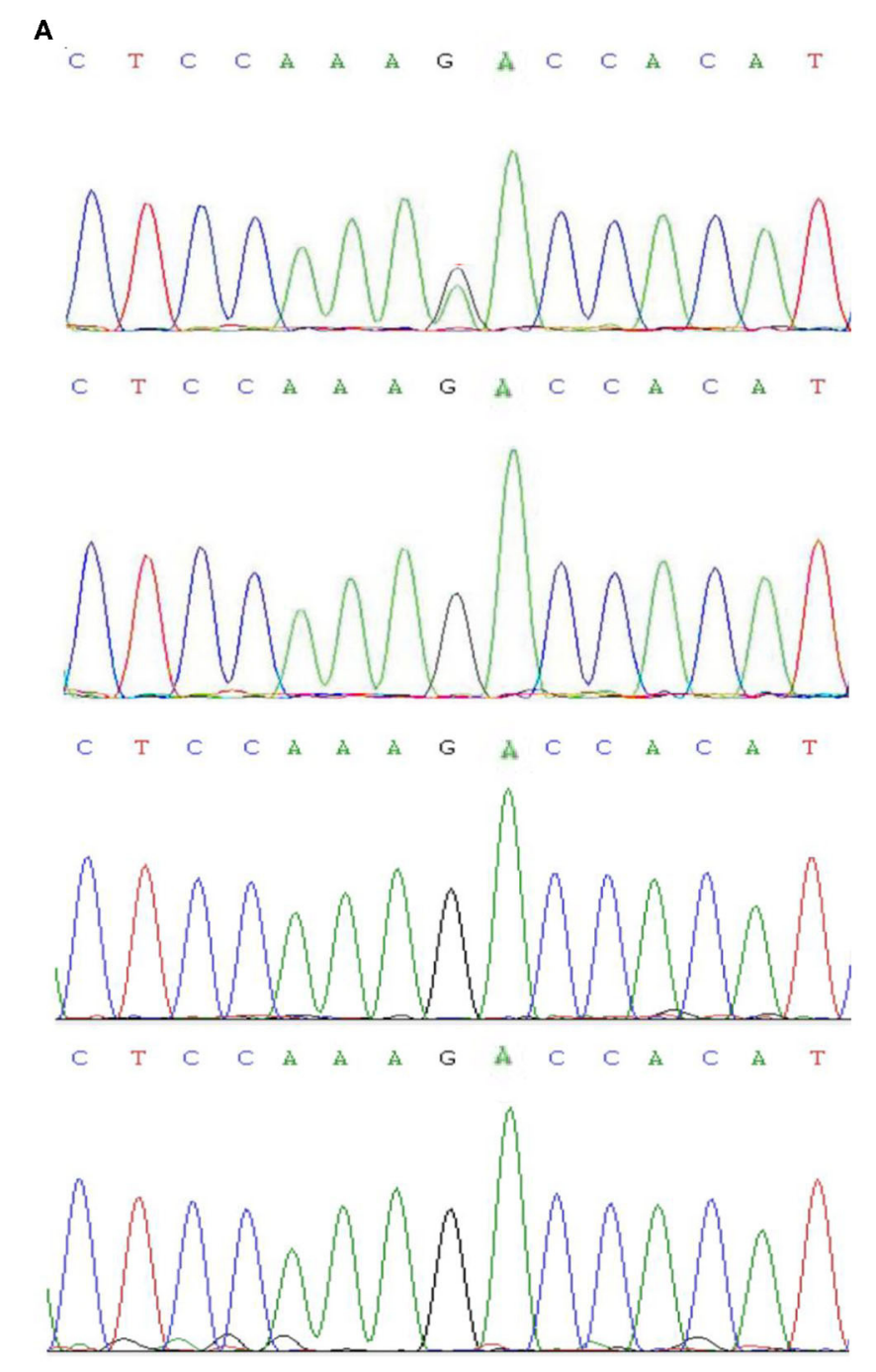

B
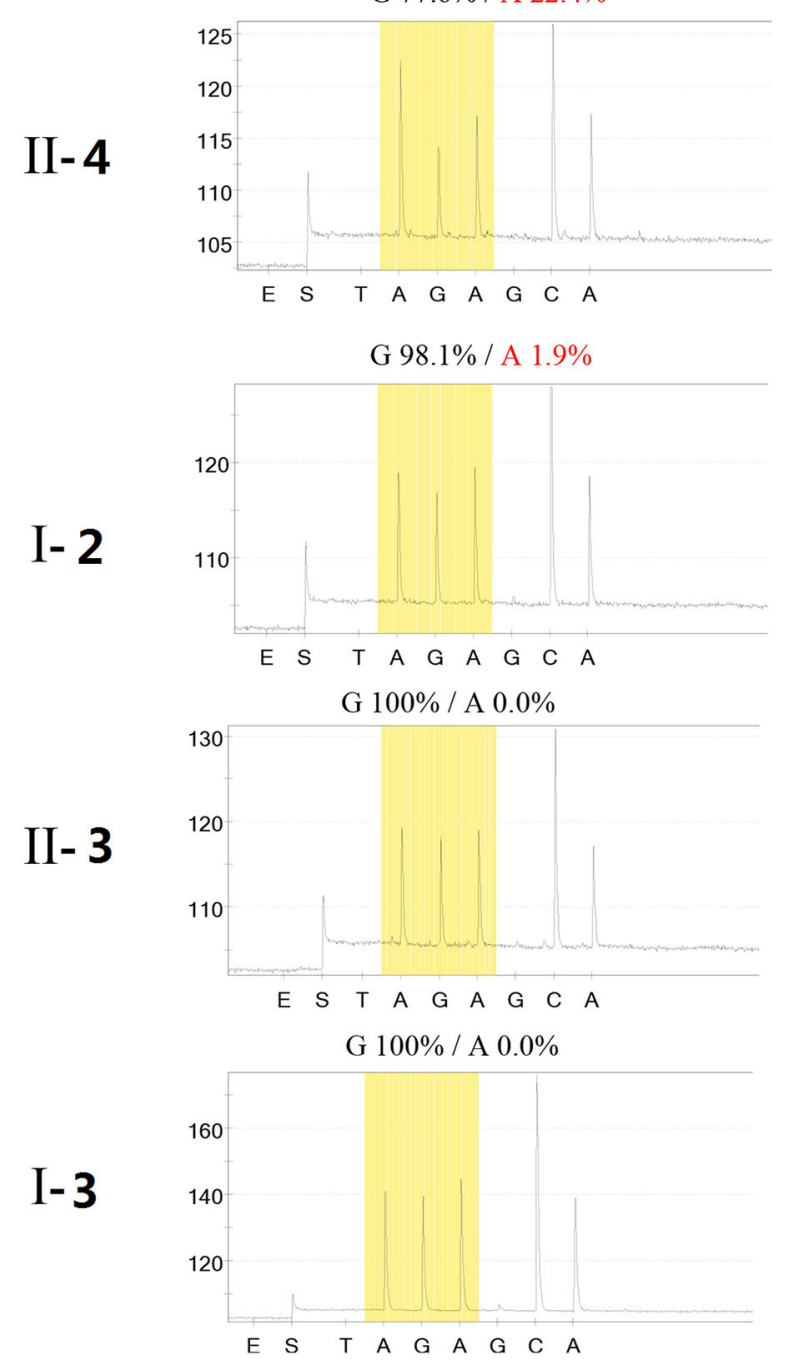

FIGURE 4 | Mutant load of the m.13513G>A mutation in this Chinese LHON family. Test results of m.13513G>A mutation in the LHON patient (II-4), his mother (I-2), his sister (II-3), and his father (I-3) assessed by next generation sequencing (A) and by pyrosequencing (B), respectively.

heteroplasmy, a severe bottleneck might abruptly transform a benign (low) frequency in a mother into a disease-causing (high) frequency in her children (26). It is also hypothesized that the drastic changes of heteroplasmy frequency between generations contribute to non-disease mutant load or higher disease severity. In this study, the LHON patient presented $22.4 \%$ mutant load, compared to his mother only $1.9 \%$ in blood cells by pyrosequencing assessment, which might be explained by the genetic drift due to germ-line bottleneck effect.

The $\mathrm{m} .13513 \mathrm{G}>\mathrm{A}$ is a point mutation in mitochondrial ND5 gene which can cause severe oxidative phosphorylation defect (26). The amino acid at position 393 of ND5 subunit encoded by m.13513 is located at an evolutionarily conserved part of a putative quinone-reactive site of the enzyme, and the D393N amino acid change caused by $\mathrm{m} .13513 \mathrm{G}>\mathrm{A}$ mutation may lead to loss of the quinine reactive site and a subsequent negative effect on the activity of the oxidative phosphorylation system, followed by significant mitochondrial impairment, as well as an increased reactive oxygen species generation and reduced ATP production, which finally resulting in the development of $\operatorname{LHON}(24,25,27)$.

In cultured cells, the threshold for m.13513G $>\mathrm{A}$ mutation causing a complex I defect is a mutant load of $\sim 30 \%$, and its impairment increased in a mutant-load dependent way $(24,28)$. The mutant load of the m.13513G $>$ A mutation which is 22.4 $\sim 33.56 \%$ in blood cells in the LHON patient in our study is consistent with above finding. Previous investigations about the mutant load in patients with $\mathrm{m} .13513 \mathrm{G}>\mathrm{A}$ mutation revealed much lower mutant load of $4 \sim 6 \%$ in blood cells, $1 \sim 5 \%$ in fibroblasts, and $13 \sim 15 \%$ in muscle in one patient, and of $11 \sim 17 \%$ in blood, hair and muscle tissues in another patient (24). Among all human tissues, optic nerve head is most susceptible to mitochondrial dysfunction, since mitochondria are most 


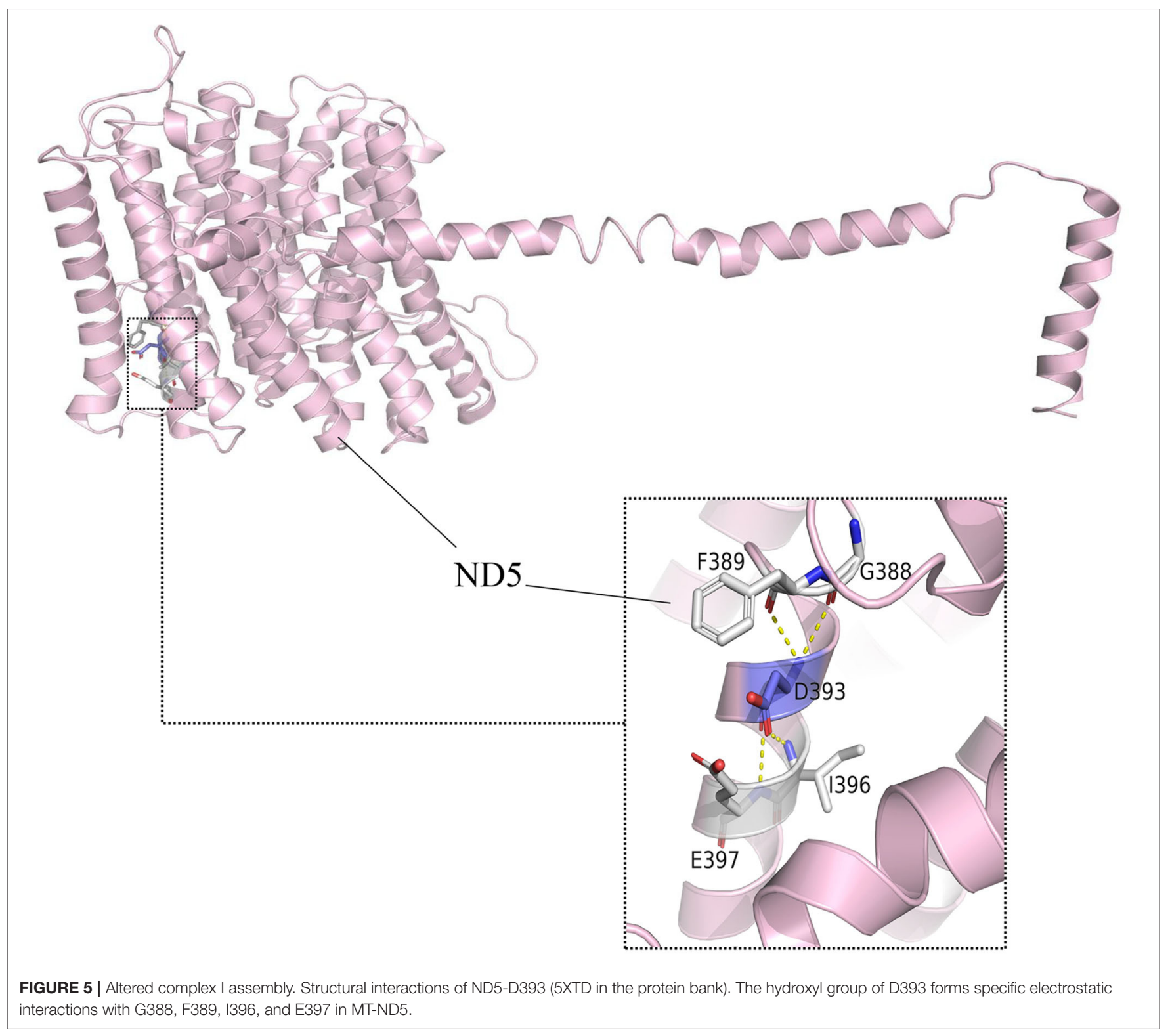

abundantly clustered in retinal nerve fibers at optic nerve head. On the other hand, previous investigations have demonstrated that ND5 synthesis is probably the rate-limiting step for the activity of complex I and consequently of respiration (24). Hence, contrary to most pathogenic mtDNA mutations which only result in the outbreak of a oxidative phosphorylation defect disease when presenting at high mutant loads in target tissues, the m.13513G $>$ A mutation may cause significant complex I defect at optic nerve head and induce an isolated LHON without any other tissue involvement even at unusually low mutant loads.

Our study revealed that the mitochondrial genomes of this pedigree belongs to the Eastern Asian haplogroups D4a. Although many other mtDNA variants were also detected in this study, there were no functionally significant mutations in this pedigree. Hence, these mtDNA variants may not have a potential modifying role in the development of visual impairment associated with m.13513G $>$ A mutation. This implied that $\mathrm{m} .13513 \mathrm{G}>\mathrm{A}$ mutation, similar to three primary mt.DNA mutations related to LHON, occurred sporadically and multiplied through evolution of the mtDNA.

There are several limitations in this study. First, only peripheral blood cells were collected for genome assessment. Considering that the percentage of $\mathrm{m} .13513 \mathrm{G}>\mathrm{A}$ mutation may vary in different tissues, it would greatly benefit if the percentage of heteroplasmy of $\mathrm{m} .13513 \mathrm{G}>\mathrm{A}$ mutation in other tissues such as urine, hair, buccal mucosa, and muscles were assessed. Unfortunately, the patient declined further genome assessment, for no other tissues except optic nerves were involved. Second, although no other ocular or extraocular pathology was found in 
TABLE 1 | mtDNA variants in a Chinese family with Leber's hereditary optic neuropathy.

\begin{tabular}{|c|c|c|c|c|c|}
\hline Gene & Position & Replacement & AA change & $\begin{array}{l}\text { Conservation } \\
\text { (H/B/M/X) }\end{array}$ & $\begin{array}{l}\text { Previously } \\
\text { reported }\end{array}$ \\
\hline \multirow[t]{11}{*}{ D-loop } & 73 & $A-G$ & & & Yes \\
\hline & 152 & $\mathrm{~T}-\mathrm{C}$ & & & Yes \\
\hline & 263 & $A-G$ & & & Yes \\
\hline & 298 & C-T & & & Yes \\
\hline & 489 & $\mathrm{~T}-\mathrm{C}$ & & & Yes \\
\hline & 514 & C-I & & & Yes \\
\hline & 515 & $A-1$ & & & Yes \\
\hline & 16092 & $\mathrm{~T}-\mathrm{C}$ & & & Yes \\
\hline & 16223 & C-T & & & Yes \\
\hline & 16274 & G-A & & & Yes \\
\hline & 16362 & $\mathrm{~T}-\mathrm{C}$ & & & Yes \\
\hline \multirow[t]{2}{*}{ 12S rRNA } & 750 & $A-G$ & & A/A/A/- & Yes \\
\hline & 1438 & $A-G$ & & $\mathrm{C} / \mathrm{C} / \mathrm{A} /-$ & Yes \\
\hline \multirow[t]{2}{*}{ 16S rRNA } & 2706 & $A-G$ & & & Yes \\
\hline & 3010 & $\mathrm{G}-\mathrm{A}$ & & & Yes \\
\hline ND1 & 3969 & C-A & & & Yes \\
\hline tRNA Gln & 4393 & C-T & & & Yes \\
\hline \multirow[t]{4}{*}{ ND2 } & 4769 & $A-G$ & & & Yes \\
\hline & 4883 & C-T & & & Yes \\
\hline & 5178 & C-A & Leu-Met & $\mathrm{L} / \mathrm{T} / \mathrm{T} / \mathrm{T}$ & Yes \\
\hline & 5231 & $\mathrm{G}-\mathrm{A}$ & & & Yes \\
\hline CO1 & 7028 & C-T & & & Yes \\
\hline ATP8 & 8414 & C-T & Leu-Phe & & Yes \\
\hline \multirow[t]{2}{*}{ ATP6 } & 8701 & $A-G$ & Thr-Ala & $\mathrm{T} / \mathrm{S} / \mathrm{L} / \mathrm{Q}$ & Yes \\
\hline & 8860 & $A-G$ & Thr-Ala & T/AVA/T & Yes \\
\hline \multirow[t]{3}{*}{$\mathrm{CO} 3$} & 9355 & $A-G$ & Asn-Ser & & Yes \\
\hline & 9380 & G-A & & & Yes \\
\hline & 9540 & $\mathrm{~T}-\mathrm{C}$ & & & Yes \\
\hline \multirow[t]{2}{*}{ ND3 } & 10398 & $A-G$ & Thr-Ala & $\mathrm{T} / \mathrm{T} / \mathrm{T} / \mathrm{A}$ & Yes \\
\hline & 10400 & C-T & & & Yes \\
\hline \multirow[t]{3}{*}{ ND4 } & 10873 & $\mathrm{~T}-\mathrm{C}$ & & & Yes \\
\hline & 11059 & C-T & & & Yes \\
\hline & 11719 & G-A & & & Yes \\
\hline \multirow[t]{4}{*}{ ND5 } & 12705 & C-T & & & Yes \\
\hline & 13104 & $A-G$ & & & Yes \\
\hline & 13513 & G-A & Asp-Asn & & Yes \\
\hline & 13708 & G-A & Ala-Thr & & Yes \\
\hline ND6 & 14668 & C-T & & & Yes \\
\hline \multirow[t]{5}{*}{ Cytb } & 14766 & C-T & Thr-lle & $\mathrm{T} / \mathrm{S} / \mathrm{T} / \mathrm{S}$ & Yes \\
\hline & 14783 & $\mathrm{~T}-\mathrm{C}$ & & & Yes \\
\hline & 15043 & G-A & & & Yes \\
\hline & 15301 & G-A & & & Yes \\
\hline & 15326 & $A-G$ & Thr-Ala & $\mathrm{T} / \mathrm{M} / \mathrm{I} / \mathrm{I}$ & Yes \\
\hline
\end{tabular}

this young-adult patient during 4 years' follow-up, other tissues involvement can not be excluded if follow-up time is much longer. Hence, regular ophthalmic and systemic examinations, as well as long-term follow-up have been highly recommended to this patient.

In summary, low mutant load of m.13513G $>\mathrm{A}$ mutation was detected in the LHON patient by both NGS and pyrosequencing of the full mt DNA genome in this study, indicating that NGS and pyrosequencing are both sensitive to detect low-level DNA mutation. Hence, patients with typical ophthalmic manifestations of LHON but with negative results of three primary point mutations related to LHON tested by targeted PCR amplification, may need to be further assessed by NGS or pyrosequencing. 


\section{DATA AVAILABILITY STATEMENT}

The raw data supporting the conclusions of this article will be made available by the authors, without undue reservation.

\section{ETHICS STATEMENT}

The studies involving human participants were reviewed and approved by Second Affiliated Hospital of Zhejiang University School of Medicine. Written informed consent to participate in this study was provided by the participants' legal guardian/next of kin.

\section{REFERENCES}

1. Rasool N, Lessell S, Cestari DM. Leber hereditary optic neuropathy: bringing the lab to the clinic. Semin Ophthalmol. (2016) 31:107-16. doi: 10.3109/08820538.2015.1115251

2. Chun BY, Rizzo JF III. Dominant optic atrophy and leber's hereditary optic neuropathy: update on clinical features and current therapeutic approaches. Semin Pediatr Neurol. (2017) 24:129-34. doi: 10.1016/j.spen.2017.06.001

3. Jurkute N, Yu-Wai-Man P. Leber hereditary optic neuropathy: bridging the translational gap. Curr Opin Ophthalmol. (2017) 28:403-9. doi: 10.1097/ICU.0000000000000410

4. Kirches E. LHON: mitochondrial mutations and more. Curr Genomics. (2011) 12:44-54. doi: 10.2174/138920211794520150

5. Yahata N, Matsumoto Y, Omi M, Yamamoto N, Hata R. TALEN-mediated shift of mitochondrial DNA heteroplasmy in MELAS-iPSCs with $\mathrm{m} .13513 \mathrm{G}>\mathrm{A}$ mutation [published correction appears in Sci Rep. (2018). Mar 13;8:4683]. Sci Rep. (2017) 7:15557. doi: 10.1038/s41598-017-15871-y

6. Brecht $M$, Richardson $M$, Taranath A, Grist S, Thorburn D, Bratkovic D. Leigh syndrome caused by the MT-ND5 m.13513G $>$ A mutation: a case presenting with WPW-like conduction defect, cardiomyopathy, hypertension and hyponatraemia. JIMD Rep. (2015) 19:95-100. doi: 10.1007/8904_2014_375

7. Pulkes T, Eunson L, Patterson V, Siddiqui A, Wood NW, Nelson IP, et al. The mitochondrial DNA G13513A transition in ND5 is associated with a LHON/MELAS overlap syndrome and may be a frequent cause of MELAS. Ann Neurol. (1999) 46:9169. doi: 10.1002/1531-8249(199912)46:6\&lt;916::AID-ANA16\&gt;3.0.CO;2-R

8. Han J, Lee YM, Kim SM, Han SY, Lee JB, Han SH. Ophthalmological manifestations in patients with Leigh syndrome. Br J Ophthalmol. (2015) 99:528-35. doi: 10.1136/bjophthalmol-2014-305704

9. Vázquez-Justes D, Carreño-Gago L, García-Arumi E, et al. Mitochondrial m.13513G $>$ A point mutation in ND5 in a 16-year-old man with leber hereditary optic neuropathy detected by next-generation sequencing. JPediatr Genet. (2019) 8:231-4. doi: 10.1055/s-0039-1691812

10. Ahmad KE, Fraser CL, Sue CM, Barton JJ. Beyond what the eye can see. Surv Ophthalmol. (2016) 61:674-9. doi: 10.1016/j.survophthal.2016.02.003

11. Chen BS, Biousse V, Newman NJ. Mitochondrial DNA 13513G >A mutation presenting with Leber's hereditary optic neuropathy. Clin Exp Ophthalmol. (2019) 47:1202-4. doi: 10.1111/ceo.13603

12. Andrews RM, Kubacka I, Chinnery PF, Lightowlers RN, Turnbull DM, Howell N. Reanalysis and revision of the Cambridge reference sequence for human mitochondrial DNA. Nat Genet. (1999) 23:147. doi: 10.1038/13779

13. Liang M, Jiang P, Li F, et al. Frequency and spectrum of mitochondrial ND6 mutations in 1218. Han Chinese subjects with Leber's hereditary optic neuropathy. Invest Ophthalmol Vis Sci. (2014) 55:1321-31. doi: 10.1167/iovs.13-13011

14. Wang J, Zhao N, Mao X, Meng F, Huang K, Dong G, et al. Obesity associated with a novel mitochondrial tRNACys 5802A $>$ G mutation in a Chinese family. Biosci Rep. (2020) 40:BSR20192153. doi: 10.1042/BSR20200131

\section{AUTHOR CONTRIBUTIONS}

$\mathrm{C}-\mathrm{bS}$ and ZL wrote and reviewed the manuscript. C-bS, H-xB, and D-nX collected the patient data. C-bS, D-nX, and QX performed literature research. C-bS, H-xB, and ZL analyzed and interpreted the clinical data. C-bS performed medical treatment. All authors read and approved the final manuscript.

\section{FUNDING}

This work was supported by Ophthalmology Star Program of Shenyang Ophthalmic Industrial Technology Institute, China (Grant Number: QMX2019-01-001).

15. Lott MT, Leipzig JN, Derbeneva O, Xie HM, Chalkia D, Sarmady $M$, et al. mtDNA variation and analysis using mitomap and mitomaster. Curr Protoc Bioinformatics. (2013) 44:1.23.1-26. doi: 10.1002/0471250953.bi0123s44

16. Ballana E, Govea N, de Cid R, Garcia C, Arribas C, Rosell J, et al. Detection of unrecognized low-level mtDNA heteroplasmy may explain the variable phenotypic expressivity of apparently homoplasmic mtDNA mutations. Hum Mutat. (2008) 29:248-57. doi: 10.1002/humu.20639

17. Ruiter EM, Siers MH, van den Elzen $C$, et al. The mitochondrial $13513 \mathrm{G}>\mathrm{A}$ mutation is most frequent in Leigh syndrome combined with reduced complex I activity, optic atrophy and/or Wolff-Parkinson- White. Eur J Hum Genet. (2007) 15:155-61. doi: 10.1038/sj.ejhg.5201735

18. Jiang $\mathrm{P}$, Jin $\mathrm{X}$, Peng $\mathrm{Y}$, Wang $\mathrm{M}$, Liu $\mathrm{H}$, Liu $\mathrm{X}$, et al. The exome sequencing identified the mutation in YARS2 encoding the mitochondrial tyrosyl-tRNA synthetase as a nuclear modifier for the phenotypic manifestation of Leber's hereditary optic neuropathy-associated mitochondrial DNA mutation. Hum Mol Genet. (2016) 25:58496. doi: $10.1093 / \mathrm{hmg} / \mathrm{ddv} 498$

19. Kong QP, Bandelt HJ, Sun C, Yao YG, Salas A, Achilli A, et al. Updating the East Asian mtDNA phylogeny: a prerequisite for the identification of pathogenic mutations. Hum Mol Genet. (2006) 15:207686. doi: $10.1093 / \mathrm{hmg} / \mathrm{ddl} 130$

20. Tanaka M, Cabrera VM, González AM, Larruga JM, Takeyasu T, Fuku $\mathrm{N}$, et al. Mitochondrial genome variation in eastern Asia and the peopling of Japan. Genome Res. (2004) 14:1832-50. doi: 10.1101/gr. 2286304

21. Bibb MJ, Van Etten RA, Wright CT, Walberg MW, Clayton DA. Sequence and gene organization of mouse mitochondrial DNA. Cell. (1981) 26:16780. doi: 10.1016/0092-8674(81)90300-7

22. Gadaleta G, Pepe G, De Candia G, Quagliariello C, Sbisà E, Saccone C. The complete nucleotide sequence of the Rattus norvegicus mitochondrial genome: cryptic signals revealed by comparative analysis between vertebrates. J Mol Evol. (1989) 28:497-516. doi: 10.1007/BF02602930

23. Roe BA, Ma DP, Wilson RK, Wong JF. The complete nucleotide sequence of the Xenopus laevis mitochondrial genome. J Biol Chem. (1985) 260:975974. doi: 10.1016/S0021-9258(17)39303-1

24. Blok MJ, Spruijt L, de Coo IF, Schoonderwoerd K, Hendrickx A, Smeets HJ. Mutations in the ND5 subunit of complex I of the mitochondrial DNA are a frequent cause of oxidative phosphorylation disease. J Med Genet. (2007) 44:e74. doi: 10.1136/jmg.2006.045716

25. Monlleo-Neila L, Toro MD, Bornstein B, Garcia-Arumi E, Sarrias A, RoigQuilis M, et al. Leigh syndrome and the mitochondrial m.13513G > A mutation: expanding the clinical spectrum. J Child Neurol. (2013) 28:15314. doi: $10.1177 / 0883073812460580$

26. Rebolledo-Jaramillo B, Su MS, Stoler N, McElhoe JA, Dickins B, Blankenberg D, et al. Maternal age effect and severe germ-line bottleneck in the inheritance of human mitochondrial DNA. Proc 
Natl Acad Sci USA. (2014) 111:15474-9. doi: 10.1073/pnas.14093 28111

27. Petruzzella V, Di Giacinto G, Scacco S, Piemonte F, Torraco A, Carrozzo R, et al. Atypical Leigh syndrome associated with the D393N mutation in the mitochondrial ND5 subunit. Neurology. (2003) 61:1017-8. doi: 10.1212/01.WNL.0000080363. 10902.E9

28. Galera-Monge T, Zurita-Díaz F, Garesse R, Gallardo ME. The mutation m.13513G $>$ A impairs cardiac function, favoring a neuroectoderm commitment, in a mutant-load dependent way. J Cell Physiol. (2019) 234:19511-2. doi: 10.1002/jcp.28549
Conflict of Interest: The authors declare that the research was conducted in the absence of any commercial or financial relationships that could be construed as a potential conflict of interest.

Copyright (C) 2021 Sun, Bai, Xu, Xiao and Liu. This is an open-access article distributed under the terms of the Creative Commons Attribution License (CC BY). The use, distribution or reproduction in other forums is permitted, provided the original author(s) and the copyright owner(s) are credited and that the original publication in this journal is cited, in accordance with accepted academic practice. No use, distribution or reproduction is permitted which does not comply with these terms. 